\section{Intermittent non-adherence with ACE inhibitor treatment and its implications for clinical trials results}

\author{
R J MacFadyen, C G Fraser, A D Struthers
}

For an individual congestive heart failure $(\mathrm{CHF})$ patient to gain maximum benefit from their angiotensin converting enzyme (ACE) inhibitor treatment, it is important for him or her to adhere fully with treatment. Many studies suggest that non-adherence with drug treatment is common in $\mathrm{CHF}$ patients and that this leads to increased hospitalisation and even death. ${ }^{1}$ Since ACE inhibitors reduce mortality, it is self evident that non-adherence will increase mortality. As CHF patients tend to be elderly and on multiple treatments, it is unsurprising that non-adherence should be a common problem in this patient group. ${ }^{2}$ However, assessing treatment adherence is notoriously difficult and all assessments of adherence are less than perfect.

In the case of adherence with an ACE inhibitor, a unique opportunity presents itself-that is, to use serum ACE measurements to assess adherence. This measurement is very robust by a variety of technologies and intrasubject variability or assay related variability is minimal $(>2 \%)$, even in the unsuppressed state. ${ }^{3}$ We have previously established that this is a viable option by showing that a

serum ACE concentration of $>20 \mathrm{u} / 1$ was $86 \%$ sensitive and $95 \%$ specific in its ability to identify non-adherence with an ACE inhibitor. ${ }^{4}$ Importantly, the dose of ACE inhibitor has virtually no impact on the ability of serum ACE to indicate ACE inhibitor non-adherence because serum ACE hits its nadir even with a tiny dose of an ACE inhibitor. ${ }^{5}$ In a cross sectional study of routine patients, we recently found that $18 \%$ of $\mathrm{CHF}$ patients exhibited $<70 \%$ adherence with their ACE inhibitor treatment. ${ }^{2}$

How variable adherence with ACE inhibitor treatment is over time within the same CHF individuals has not previously been assessed. This is particularly relevant to the interpretation of the results for patients taking part in long term clinical mortality trials since nonadherence with treatment could easily lead to a false negative result or more likely an under appreciation of treatment effect at a variety of levels, whether symptoms, morbidity (hospitalisation rates) or mortality.

\section{Methods and results}

A cohort of $32 \mathrm{CHF}$ patients was followed for one year using monthly visits by a research
Cardiac Unit (Floor 7), Highland Acute Hospitals, Raigmore Hospital, Old Perth Road, Inverness IV2 3RE, UK

R J MacFadyen

Department of Clinical Pharmacology \& Therapeutics, and Biomedical Medicine ${ }^{\star}$, Ninewells Hospital Medical School Dundee DDI 9SX, UK C G Fraser*

A D Struthers

Correspondence to: Dr MacFadyen, robert.macfadyen@raigmore. scot.nhs.uk

Accepted 25 October 2000

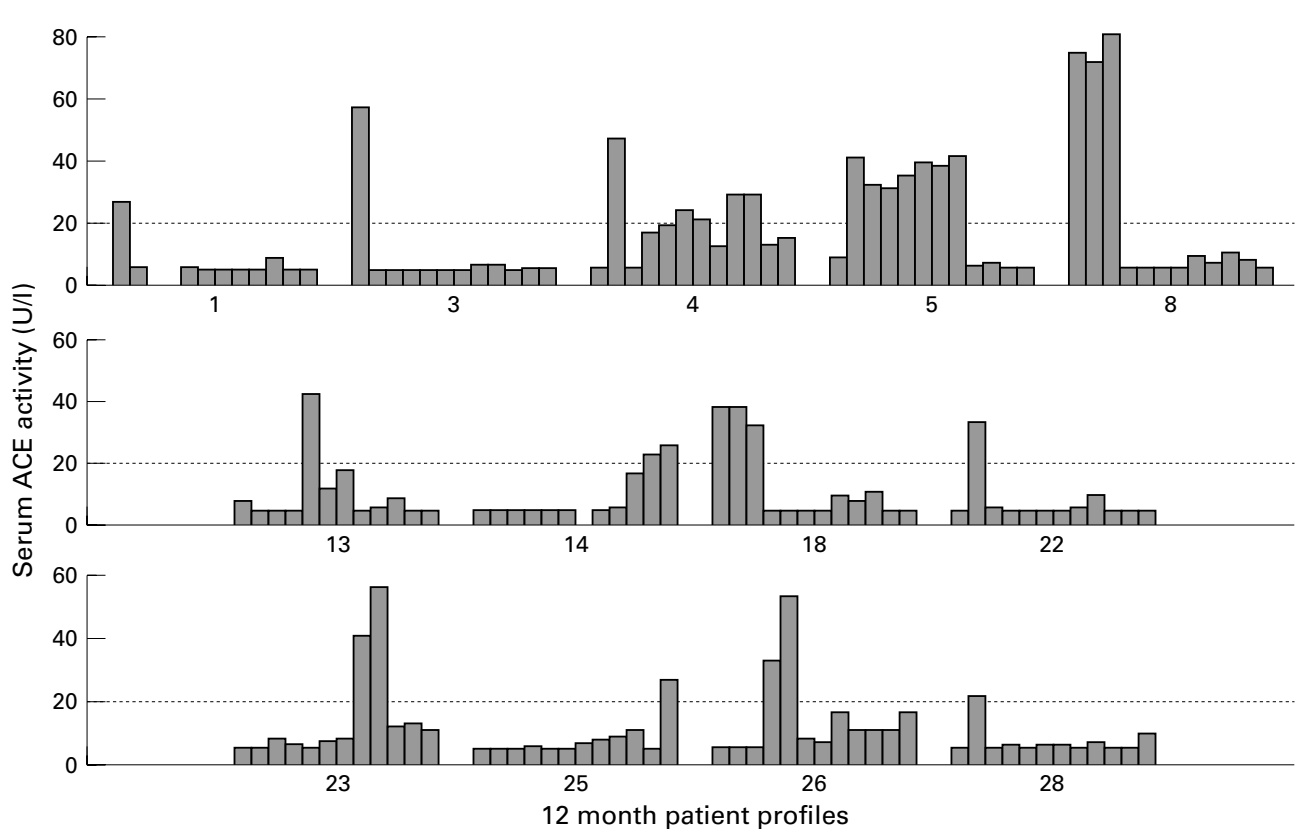

Figure 1 Sequential measurement of serum ACE activity over a 12 month period. For clarity, all that is shown are the profiles of the 13 patients in whom serum ACE was $>20$ u/1 during at least one time point. 
nurse as part of a larger research project. They were all taking chronic frusemide (furosemide) and non-captopril ACE inhibitor treatment, and were supposedly ingesting the same dose for the whole year which was verbally confirmed at each visit during review of all prescribed and purchased medicines. Patients taking captopril were excluded from this analysis as captopril has a poor affinity for ACE in vitro, making deferred serum ACE measurements inaccurate. At each visit the study nurse took blood that was subsequently assayed for serum ACE.

Three hundred and eighty samples for serum ACE were taken over one year (interassay variation $<10 \%)$. Of these samples, $30(8 \%)$ had serum ACE $>20 \mathrm{u} / 1$ at some point during follow up (fig 1). These high values for serum ACE were concentrated in 13/32 individuals $(41 \%)$; the number of samples with a serum ACE $>20 \mathrm{u} / 1$ varied from 1 to 7 out of a possible total of 12 (median value 2 ). Since these were patients who volunteered to take part in a research study, one might expect that their levels of adherence would be better than routine elderly CHF patients. Our earlier work suggests that serum ACE only becomes $>20 \mathrm{u} / 1$ when the ACE inhibitor has not been taken for $>36$ hours. Since the patients were stable and supposed to be taking the same dose of the same ACE inhibitor for the full year, the intermittent intra-individual increases in serum ACE almost certainly reflect intermittent non-adherence with ACE inhibitor treatment over time. The patterns seen within subjects show that this is not only common but that it was typically intermittent and total nonadherence to prescribed ACE inhibitor treatment was rare.

\section{Discussion}

Our study shows that there is both large interindividual and large intra-individual variability in adherence to ACE inhibitor treatment, which presents a practical everyday problem to the clinician. That problem is how to identify whether an individual patient is adherent with his or her medication or not. This study suggests that identifying such individuals using serum ACE measurement is possible. It is reasonable to suggest that improving their adherence might well improve clinical outcome, which is particularly relevant given the massive costs of treatment failure in this group. ${ }^{6}$ This might often be by the simple step of correcting confusion over their various tablets. This needs to be tested in a randomised controlled trial.

The intermittent non-adherence that we saw in patients taking part in research is also very important as part of the interpretation of clinical trials. Divergent results in apparently similar circumstances might be explained by variable adherence. In clinical trials involving ACE inhibitors this could easily be done by monitoring serum ACE, although this has never been done in any of the large clinical trials of ACE inhibitors.

1 Ghali JK, Kadakis S, Cooper R, et al. Precipitating factors leading to decompensation of heart failure. Arch Intern Med 1988;148:2013-6.

2 Struthers AD, Anderson G, MacFadyen RJ, et al. Nonadherence with ACEI treatment is common in heart failure and can be detected by routine serum ACE activity assays. Heart 1999;82:584-8.

3 Fogarty Y, Fraser CG, Browning MCK. Intra and inter individual varibility of serum angiotensin converting enzyme activity: clinical implications. Ann Clin Biochem 1989;26:201-2.

4 Struthers AD, MacFadyen RJ, Fraser C, et al. Nonadherence with ACE inhibitor therapy: a comparison of different ways of measuring it in patients with CHF. $\mathcal{F} \mathrm{Am}$ Coll Cardiol 1999;34:2072-7.

5 van Veldhuisen DJ, Genth-Zotz S, Brauower J, et al. High versus low dose ACE inhibition in CHF. $7 \mathrm{Am}$ Coll Cardiol 1998;32:1811-8.

6 Andersson F, Cline C, Ryden-Bergsten T, et al. Angiotensin converting enzyme (ACE) inhibitors and heart failure - the consequences of underprescribing. Pharmacoeconomics 1999;15:535-50. 